\title{
Identification of Cleavage Origins Using Focused Ion Beam (FIB) Sectioning
}

\author{
S. Xu*, R. Bouchard*, J. Li** and W.R. Tyson* \\ * CANMET-Materials Technology Laboratory, 568 Booth St., Ottawa, ON, Canada K1A 0G1 \\ ** Fibics Inc, 556 Booth St. Suite 200, Ottawa, ON, Canada K1A 0G1
}

The most common mechanism of brittle fracture in steels is cleavage, which involves atomic bond breakage along mainly $\{001\}$ crystal planes. Cleavage fracture initiates from a single or multiple origins and often spreads across the whole structure after initiation. In order to control cleavage fracture, it is first necessary to identify the microstructural features where cleavage originates. Determination of the position and geometry (size and shape) of cleavage origins can, by using the methods of fracture mechanics, provide quantitative information related to the fracture process such as the cleavage stress and the effective surface energy.

Cleavage initiation sites can be identified by tracing back "chevron markings" at low magnifications and "river markings" at high magnifications. In steel welds, cleavage origins have been shown in the literature to be often associated with nonmetallic inclusions. [1,2] Cleavage origins have also been associated with TiN precipitates in microalloyed steels [3] and with inclusions or carbide particles in C/Mn steels. In modern plate/linepipe steels, the levels of impurities ( $\mathrm{S}$ and $\mathrm{P}$ ) and $\mathrm{C}$ have been substantially reduced. Therefore, there are not as many inclusions and carbides in these steels to initiate cleavage, and other mechanisms may become important.

FIB systems have been used intensively in the electronics industry and have now been developed into a general materials science tool. [4] FIB technology is promising for the identification of cleavage origins because of its capacity for precision sectioning (precise stage movement and tilt, stage stability, ion source stability, and ion beam current adjustment), imaging sensitivity to composition and orientation, and possibility for in-situ examination after sectioning and ionpolishing.

In this work, cleavage origins were identified using scanning electron microscopy (SEM) and were then sectioned and examined by the FIB technique. The samples shown here were tested in a project to study cleavage toughness variability at CANMET/MTL. Figures 1 and 2 show SEM fractographs of cleavage initiation sites of samples of a low-C plate steel. The SEM fractographs do not reveal any clear microstructural features other than possible grain boundary intersections associated with the cleavage origins. The FIB system was employed to section through the cleavage origins to reveal microstructural features underlying them. The broken lines in Figs. 1 and 2 indicate the approximate locations subsequently cross-sectioned by the ion beam. Figures 3 and 4 show the FIB secondary electron images at areas beneath the cleavage origins. Before FIB sectioning, tungsten was deposited on the fracture surface to prevent edge rounding during sectioning; the tungsten layers are seen in the top part of Fig. 3. Figure 3 shows that the cleavage origin in this sample was at the pearlite-ferrite boundary or in the pearlite. Figure 4 reveals that the cleavage origin was at a ferrite grain boundary in this case; the dark contrast close to the origin in the ferrite grains may be indicative of plastic deformation. [5]

This is the first time to the authors' knowledge that the FIB technique has been applied to the study of cleavage fracture. The results show that cleavage is not necessarily initiated by inclusions or 
large carbides but that in modern steels cleavage could initiate at other locations such as grain boundaries, pearlite/ferrite boundaries or pearlite.

\section{REFERENCES}

1. J.H. Tweed and J.F. Knott, Met. Sci. 17 (1983) 45.

2. W.R. Tyson and B. Marandet, ASTM STP 945, ASTM, Philadephia, PA (1988) 19.

3. A. Echeverria et al., Inter. J. Fract. 89 (1998) L39.

4. M.W. Phaneuf, Micron 30 (1999) 277.

5. M.G. Burke et al., Microsc. Microanal. 6 (Suppl 2: Proceedings) (2000) 530.

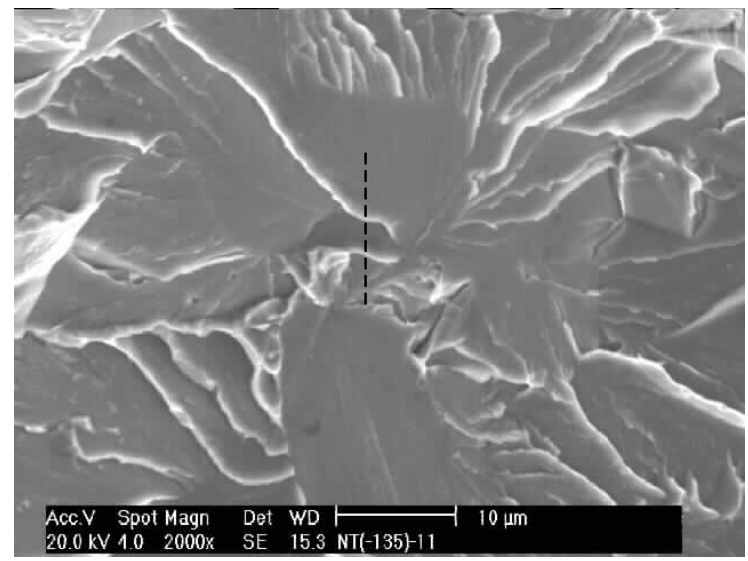

FIG. 1. SEM fractograph showing the cleavage origin of a notched tensile sample tested at $-135^{\circ} \mathrm{C}$

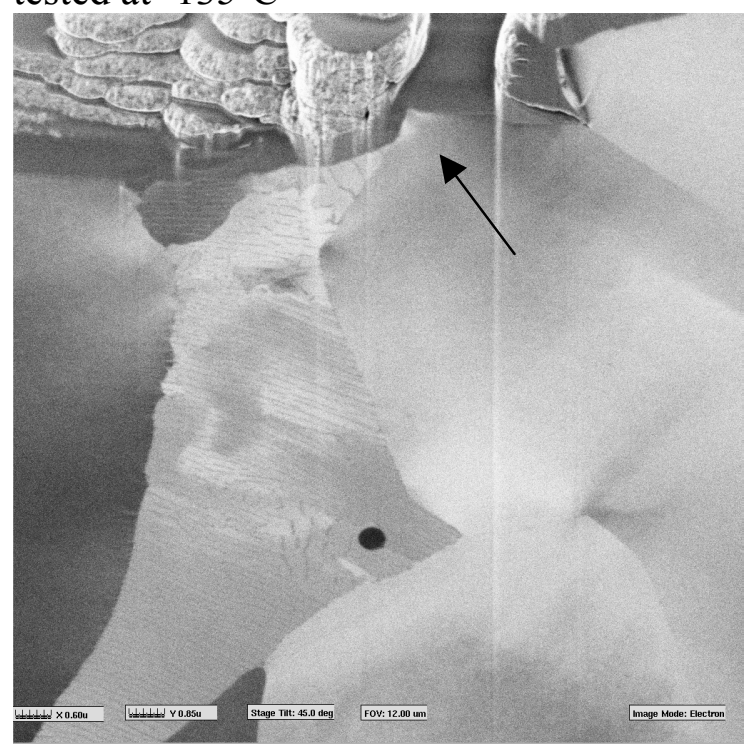

FIG. 3. FIB secondary electron micrograph beneath the cleavage origin in Fig. 1. The arrow indicates the origin. The photo was tilted $45^{\circ}$ along the $\mathrm{Y}$-axis. Original magnification: $\mathrm{X}: \times 5433, \mathrm{Y}: \times 3835$.

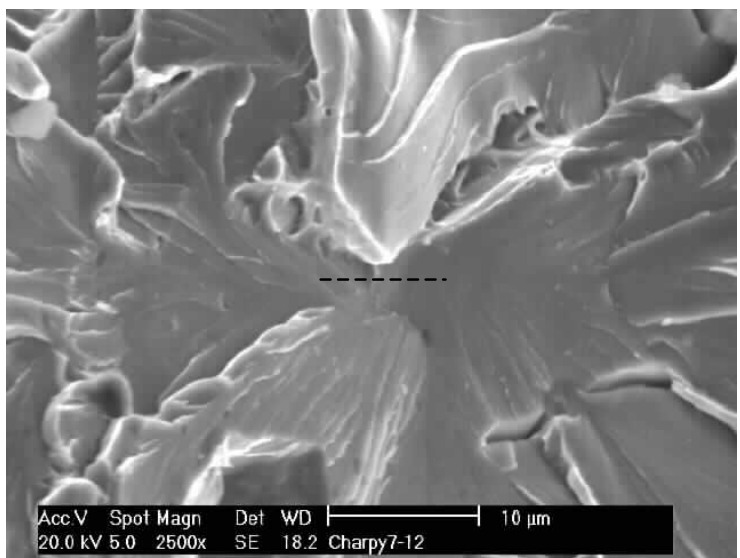

FIG. 2. SEM fractograph showing the cleavage origin of a Charpy V-notch sample tested at $-80^{\circ} \mathrm{C}$

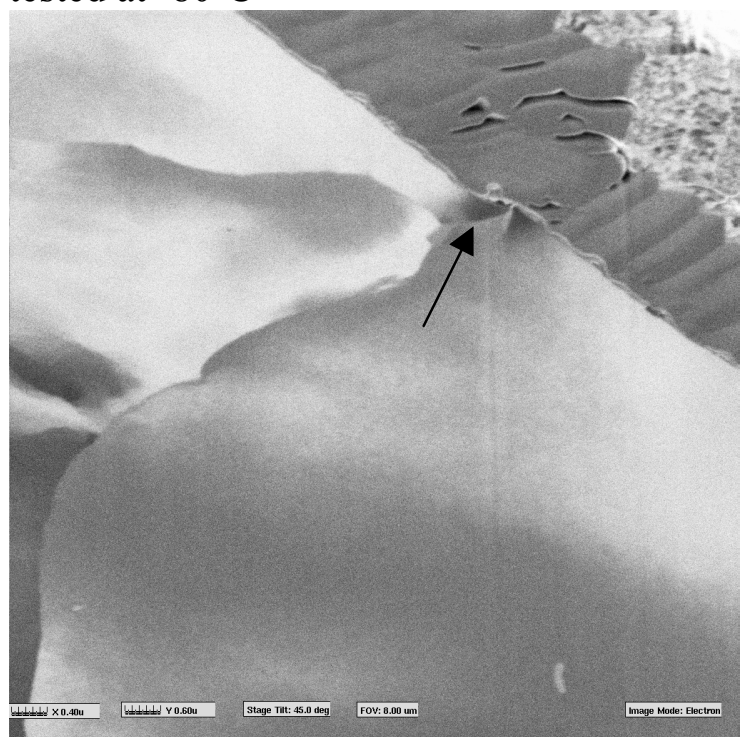

FIG. 4. FIB secondary electron micrograph beneath the cleavage origin in Fig. 2. The arrow indicates the origin. The photo was tilted $45^{\circ}$ along the $\mathrm{Y}$-axis. Original magnification: $\mathrm{X}: \times 8150, \mathrm{Y}: \times 5433$. 